\title{
X-Ray Diffraction Measurements and Investigation of the Stress Relaxation in Autofrettaged AISI 4140 Steel Thick Walled Cylinders
}

\author{
N. Lyubenova ${ }^{1, a^{*}}$, J. Pineault ${ }^{2, b}, H$. Brünnet ${ }^{1, c}, D$. Bähre ${ }^{1, d}$ \\ ${ }^{1}$ Saarland University, Institute of Production Engineering, Campus A4.2, Saarbrücken, \\ Germany \\ ${ }^{2}$ Proto Mfg. Inc., 12350 Universal Drive, Taylor, Michigan, USA

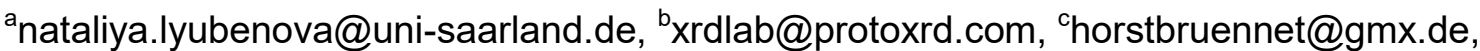 \\ d.baehre@mx.uni-saarland.de
}

\begin{abstract}
Keywords: Residual Stress, Autofrettage, Post-Machining, X-Ray Diffraction, Stress Correction
\end{abstract}

\begin{abstract}
Hydraulic autofrettage is a manufacturing process that induces favorable compressive residual stresses and is especially suitable for the treatment of internally pressurized components. If autofrettage is not the final treatment applied, the application of post-machining or other cold working processes can lead to a relaxation and redistribution of the stresses induced by the autofrettage process. In this paper, comprehensive X-ray diffraction residual stress measurements were performed and the influence of the applied autofrettage pressure and post-machining on the resultant residual stress vs. depth profiles was investigated.
\end{abstract}

\section{Introduction}

It is well known that compressive residual stresses are favourable because they act to close existing cracks in components and prevent the generation of new ones. When a component experiences inservice loading, applied tensile stresses will be shifted by the compressive residual stress (RS) field, if present. If the compressive RS field is of sufficient magnitude, the final stress state may still remain locally compressive despite the superimposed applied tensile stresses. There are several processes that are able to induce high magnitude compressive RS; e.g. shot peening, deep rolling and laser shock peening. The autofrettage (AF) process is especially suitable for treating internal geometries, e.g. components of the common rail diesel injection system. It leads to a beneficial and pronounced compressive RS vs. depth profile $[1,2]$ and several authors report an extension in fatigue life for components treated with this process [3, 4]. Its principle can be explained as follows [5]: when applying AF, a low-viscosity hydraulic medium is used to rapidly over-pressurize the treated component. If the resulting stresses exceed the yield strength of the material, then elasto-plastic deformation will result. Typically, the inner surface of the treated component deforms plastically while the outer surface of the component remains only elastically deformed. After releasing the AF pressure, the elastically deformed region of the component strives to return to its original state but is prevented from doing so by the inner plastically deformed region. This inhomogeneous deformation leads to the generation of compressive RS on the inner region of the component. This compressive region is compensated for with tensile stresses on the outer region of the component. The AF pressure is the most important processing input parameter and changing it leads to different RS vs. depth profiles in the part [6]. It has been shown that the AF process not only induces RS but also results in concomitant macroscopic shape deviations [7]. When high dimensional accuracy of the treated component is required, it may be necessary to perform a post-machining operation that could result in a redistribution and/or relaxation of the RS induced by the AF process. As such, the following paper presents investigations that include: pre-machining, autofrettage and post-machining. 
X-ray diffraction (XRD) is an established, generally applicable, and time-proven method for near surface (up to $0.025 \mathrm{~mm}$ ) RS measurements [8]. Accompanied with an electropolishing procedure, it offers the possibility to investigate RS at depth. In the presented paper, XRD techniques were employed to measure the RS vs. depth profiles in thick walled cylinders that were treated with different AF pressures and partially post-treated using a reaming operation. Due to the geometric constraints inherent to the specimens, they were sectioned in half to enable XRD based RS measurements on their inner diameter (ID). To account for the relaxation and redistribution of the RS induced by the AF process as a result of axial sectioning, strain gauges were applied to the inner and outer diameters (OD) of the specimens' surfaces prior to cutting. The strain gauges were then monitored during the sectioning process and the results obtained were used to correct the measured stresses for the effect of sectioning. Since the XRD technique samples a relatively thin surface layer, material removal techniques were applied so as to obtain the full through wall thickness RS profile. To this end, electropolishing techniques were employed and the resulting stress relaxation was corrected for using the analytical methods proposed by Moore and Evans [9].

\section{Experimental setup}

The samples used for the experiments are thick walled cylinders, manufactured from high strength AISI 4140 steel (hardened at $840{ }^{\circ} \mathrm{C}$ and tempered at $610^{\circ} \mathrm{C}$ ). Two measurement points (MP1 and MP2) were chosen to characterize the different treatment sequences on both sides of the thick walled cylinders. The process and measurement chains are shown in Table 1.

Table 1 - Process and measurement chains for the thick walled cylinders

\begin{tabular}{|c|c|c|c|c|c|}
\hline MP1 & MP2 & Process and measurement $\mathrm{c}$ & chain & Process parameters & Residual Stresses \\
\hline+ & + & & Raw part & $\begin{array}{l}\text { Rolled } \\
\text { Hardened at } 840^{\circ} \mathrm{C} \\
\text { Tempered at } 610^{\circ} \mathrm{C}\end{array}$ & $\begin{array}{l}\text { Present, unknown } \\
\text { Randomly distributed }\end{array}$ \\
\hline+ & + & & $\begin{array}{l}\text { Pre- Machining } \\
\text { (boring) }\end{array}$ & $\begin{array}{l}\text { HSS twist drill } \\
\text { Length: } 125 \mathrm{mmm} \\
\mathrm{n}=2001 / \mathrm{min}, \mathrm{v}_{\mathrm{f}}=1 \mathrm{~mm} / \mathrm{s} \text {, } \\
\mathrm{ID}=\varnothing 9 \mathrm{H} 12\end{array}$ & 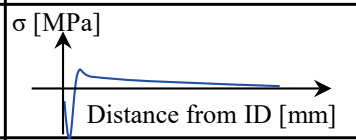 \\
\hline+ & + & & Autofrettage & $\begin{array}{l}\text { Autofrettage pressure levels: } \\
\mathrm{P}=2000,8000,8500 \\
9000 \text { and } 9500 \text { bar }\end{array}$ & $\stackrel{\uparrow_{\text {Distance from ID [mm }]}^{\sigma[\mathrm{MPa}]}}{\longrightarrow}$ \\
\hline$=$ & + & & $\begin{array}{l}\text { Post-Machining } \\
\text { (reaming) }\end{array}$ & $\begin{array}{l}\text { HSS }- \text { E } 6 \text { blades reamer } \\
\mathrm{n}=2001 / \mathrm{min} \\
\mathrm{vf}=0.6 \mathrm{~mm} / \mathrm{s} \\
\text { OD }(\text { up to middle })=\varnothing 10 \mathrm{H} 7\end{array}$ & $\underset{\text { Distance from ID [mm] }}{\stackrel{\sigma}{\sim} \text { Stress relaxation? }}$ \\
\hline+ & + & & $\begin{array}{l}\text { Strain gauges } \\
\text { application }\end{array}$ & N.D. & Unchanged \\
\hline+ & + & & $\begin{array}{l}\text { Axial cutting/ } \\
\text { strain gauges } \\
\text { reading }\end{array}$ & N.D. & 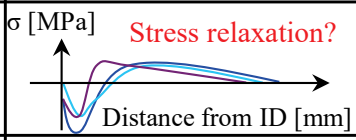 \\
\hline+ & + & & \begin{tabular}{|c|} 
XRD \\
Measurements/ \\
Electropolishing \\
\end{tabular} & See Table 2 & 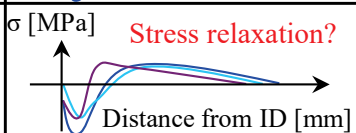 \\
\hline
\end{tabular}

Samples were prepared by first boring $\left(n=2001 / \mathrm{min}, \mathrm{v}_{\mathrm{f}}=1 \mathrm{~mm} / \mathrm{s}\right)$ an inner diameter of $9 \mathrm{~mm}$, using a HSS twist drill. The cylinders were then treated with AF pressures ranging from 2000 to 9500 bar. The treatment with 2000 bar did not lead to a plastification of the cylinders' surface and RS was therefore not generated; the cylinders treated with an AF pressure of 2000 bar are thus considered to be untreated. A reaming operation $\left(n=2001 / \mathrm{min}, \mathrm{v}_{\mathrm{f}}=0.6 \mathrm{~mm} / \mathrm{s}\right)$ to an inner diameter of $10 \mathrm{~mm}$ was then performed on the right side of the cylinder leaving the left half of each cylinder 
(MP1) bored and autofrettaged, whereas the right half (MP2) was additionally reamed. The reason for this additional treatment was to investigate the RS relaxation and redistribution due to this postAF treatment. Strain gauges were then applied to the inner and the outer surfaces of the specimens and an axial cut provided access for XRD RS measurements on the inner diameter of the specimens. After the axial cut was performed, the stress relaxation on both the inner and outer diameters of each specimen was calculated via the strain gauge readings. For the XRD RS measurements the setup parameters shown in Table 2 were employed.

Table 2 - X-ray diffraction setup Parameters

\begin{tabular}{|l|l|l|}
\hline Instrument: Proto LXRD & Crystallographic Plane: $\{211\}$ & Oscillation(s): Beta $4.00^{\circ}$ \\
\hline $\begin{array}{l}\text { Software Version: XRDWIN 2.0 } \\
\text { Build } 86\end{array}$ & Bragg Angle $(2 \theta): 156.4^{\circ}$ & $\begin{array}{l}\text { Collection Time: } 0.25 \text { seconds x } 20 \\
\text { exposures }\end{array}$ \\
\hline Goniometer Configuration: Psi & Aperture: $2 \mathrm{~mm}$ & Peak Fit: Gaussian $85 \%$ \\
\hline Target: $\mathrm{Cr}\left(\mathrm{K} \alpha_{\text {avg } 2.291}\right.$ Angstroms $)$ & Psi Tilts: 22 & LPA Correction On: Yes \\
\hline Target Power: $30 \mathrm{kV}, 25 \mathrm{~mA}$ & $\begin{array}{l}\text { Tilts: }\left(0^{\circ}, \pm 25.00^{\circ}, \pm 20.60^{\circ}, \pm 15.85^{\circ},\right. \\
\left. \pm 11.80^{\circ}, \pm 3.74^{\circ}\right)\end{array}$ & Background Subtraction: Linear \\
\hline
\end{tabular}

The RS vs. depth profile was generated by means of sequential XRD measurements and material removal using electropolishing techniques to mid-wall thickness from the ID. RS measurements were then performed from the OD to mid-wall thickness. Once the RS measurements were completed, the pertinent data corrections were applied. First, the gradient correction was applied as per SAE HS-784 [10]. This correction accounts and corrects for steep RS gradients and their effect on sampling depth variations with incident x-ray beam angle. Next, the material removal correction was applied to the gradient corrected data; this correction is based on the analytical methods proposed by Moore and Evans [9] for cylinders and plates and is still the most widely used correction in industry. Finally, the measurement results obtained were corrected for stress relaxation due to sectioning using the strain gauge data. The two data sets obtained on the OD to mid-wall thickness and ID to mid-wall thickness were combined into one contiguous through wall RS profile.

\section{Results}

XRD measurements were performed for the untreated (AF 2000 bar) as well as the specimens treated with $8000,8500,9000$ and 9500 bar AF pressure. In order to characterize statistical variability, three different specimens treated with the same AF pressure (8000 bar) were characterized using XRD. In Fig. 1, the residual hoop stress vs. depth for these specimens is plotted after gradient, depth and strain corrections were applied. The results for the bored and AF side (MP1) of the AF 8000 bar specimens plotted in Fig. 1a), indicate that the maximum induced RS for each specimen is just below the surface and ranges from -450 to $-550 \mathrm{MPa}$ in compression. It can also be seen that the RS becomes slightly tensile at a depth of approximately $2 \mathrm{~mm}$. On the outer diameter of the specimens, a thin layer of compressive RS can be also observed which is thought to be due to the turning operation that was used for their pre-machining. Fig. 1b) depicts the hoop RS for the specimens autofrettaged with 8000 bar on the bored, autofrettaged, and reamed side of the specimen (MP2). The maximum induced compressive RS is lower in magnitude and on the order of $-400 \mathrm{MPa}$. This reduction in $\mathrm{RS}$ is attributed to the reaming operation after the AF which removes a part of the most compressive loaded material thus leading to a redistribution and relaxation of the balancing tensile RS towards the OD. Brünnet et al. [7] investigated the influence of the post-machining, namely the reaming operation after AF, and confirms that it leads to a relaxation and redistribution of RS. 

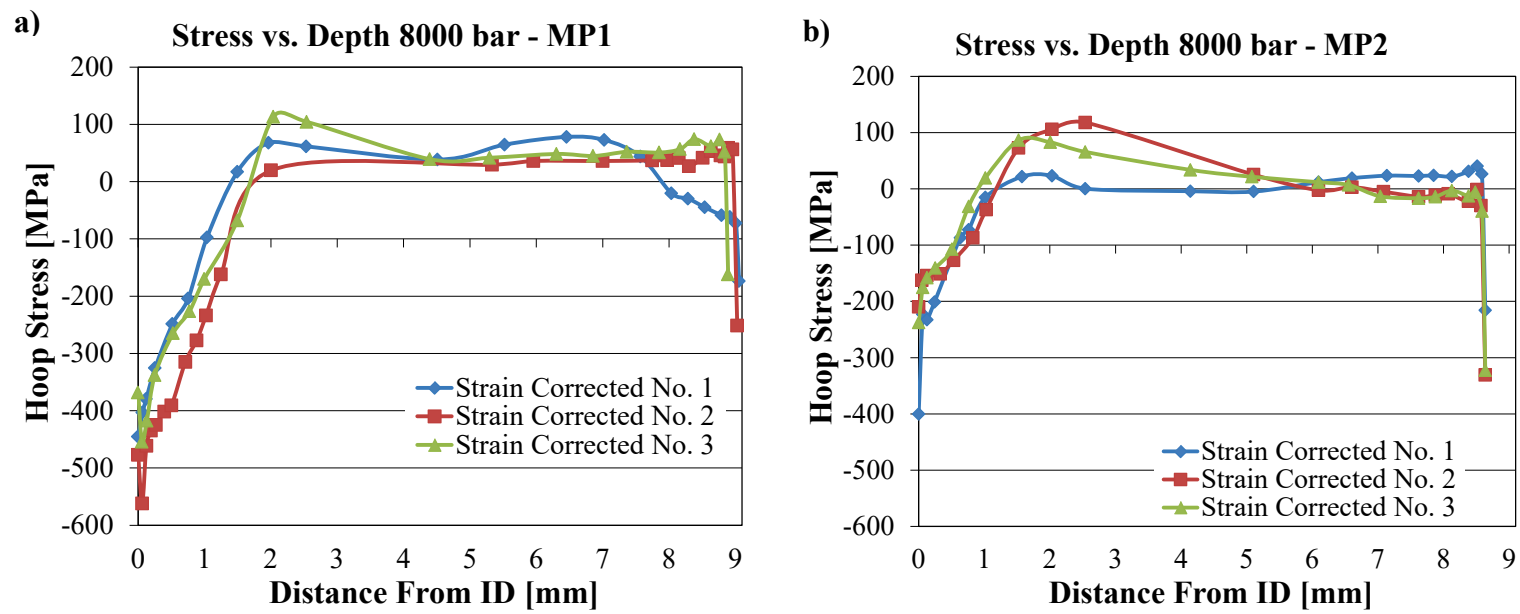

Fig. 1 -Corrected hoop RS for specimens AF treated at 8000 bar-a) at MP1 and b) at MP2.

The hoop RS vs. depth profile for the non-autofrettaged specimen can be seen in Fig. 2. On the bored side of the specimen (MP1), see Fig. 2a), a thin layer of compressive RS is visible near the ID and the OD. This can be attributed to the boring (ID) and turning (OD) operations. In Fig. 2b), the stress profile for the reamed side of the specimen (MP2) is plotted. It can be seen that the near surface measured RS was approximately 70 to $100 \mathrm{MPa}$ less compressive as compared to MP1. This indicates that the reaming operation itself results in lower magnitude compressive RS as compared to the boring operation.
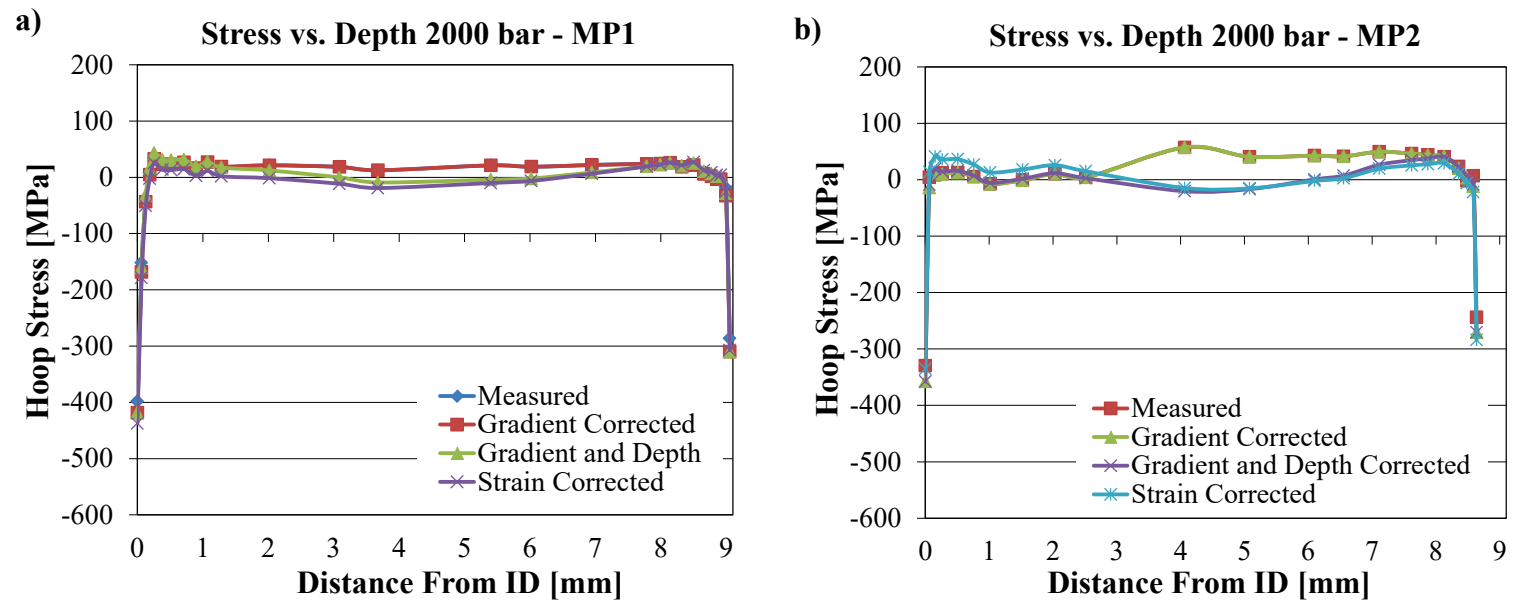

Fig. 2 - Hoop RS for specimens AF treated at 2000 bar - a) at MP1 and b) at MP2.

The hoop RS vs. depth profiles measured at MP1 for the specimens treated with different AF pressures are plotted in Fig. 3. Fig. 3a) depicts the "as measured" results. Here, it can be observed that the non-autofrettaged specimen (2000 bar) has a very thin layer (about $0.5 \mathrm{~mm}$ ) of compressive $\mathrm{RS}$ on the ID that is produced during the boring operation. An unanticipated result was observed in the curves for the specimens that were autofrettaged at different pressures. It was expected that increasing the AF pressure would lead to a greater maximum induced compressive RS. Instead, the apparent maximum stresses remained approximately the same with increasing AF pressure and in some cases, it decreased. Nevertheless, the higher AF pressures produced a deeper compressive RS layer that was compensated for with higher subsurface tensile stresses. When examining the corrected results, see Fig. 3b), it is clear that even after the corrections are applied, the maximum compressive RS achieved does not increase with increasing AF pressure, and in some cases, it decreases slightly. If the difference in the RS vs. depth profiles for the three specimens treated with 
an AF pressure of 8000 bar is considered (about $75-100 \mathrm{MPa}$ ) it can be concluded that, in the AF pressure range (8000 to 9500 bar), the measured RS is of similar magnitude.

a)

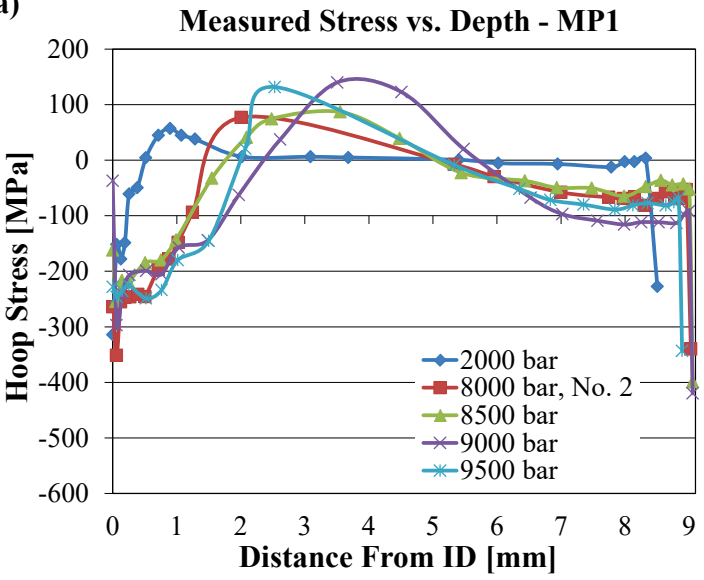

b)

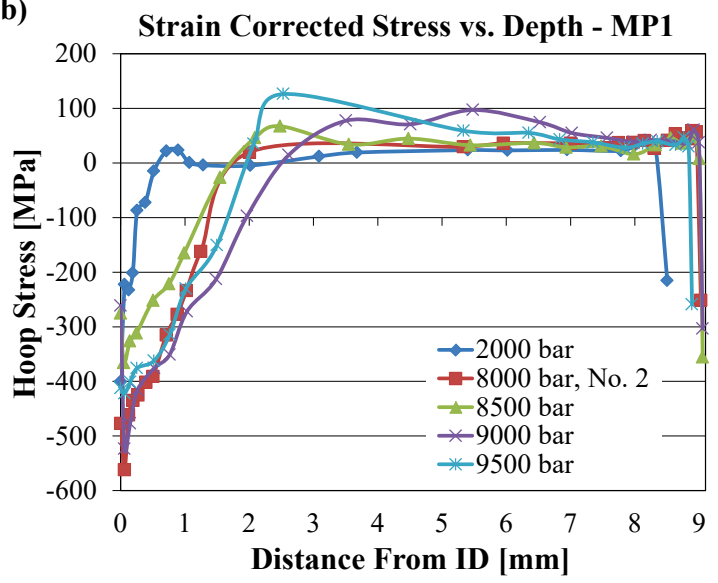

Fig. 3 - Hoop RS AF variation at MPI - a) As measured and b) Gradient, depth and strain corrected.

The RS vs. depth profiles for the specimens treated with different AF pressures at MP2 (boring, $\mathrm{AF}$ and consecutive reaming) are plotted in Fig. 4. The "As measured" profiles can be seen in Fig. 4a). Here, it can be observed that material removed by the reaming operation leads to a significant relaxation in the compressive RS induced by the AF operation. When compared to results obtained at MP1 (where the AF process represents the final treatment applied), results obtained in the compressive RS region near the ID at MP2 are more than $100 \mathrm{MPa}$ less compressive. It can also be observed that higher magnitude applied AF pressures result in higher magnitude stress relaxation. Notwithstanding, the apparent depth of the induced compressive RS remains unaffected. The "Gradient, depth and strain corrected" RS vs. depth profiles at MP2 for the different applied AF pressures can be seen in Fig. 4b). The corrections applied to the data lead to a shift, similar to the one observed at MP1, for all of the stress curves, most noticeably in the compressive region near the ID. The maximum stress relaxation induced by the reaming operation was about $200 \mathrm{MPa}$ (see Fig. 3b) as a point of comparison to MP1). It can also be observed that the depth of the compressive RS layer increases by a few tenths of a millimeter as compared to the uncorrected data.

a)

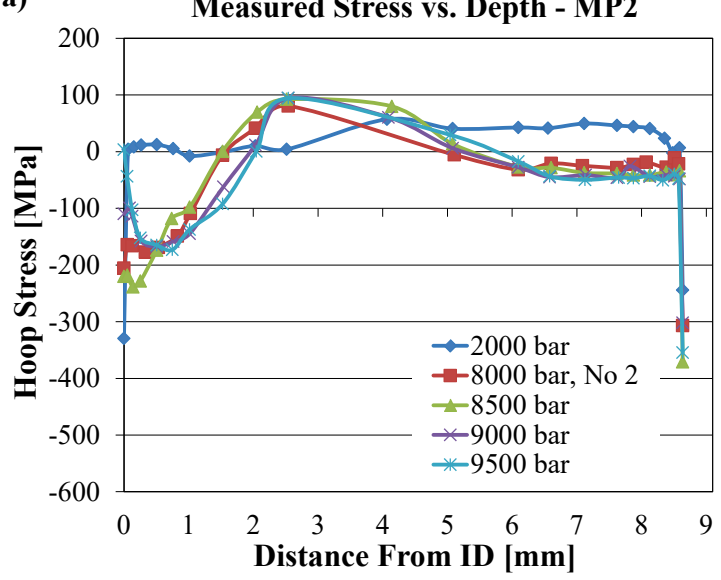

b)

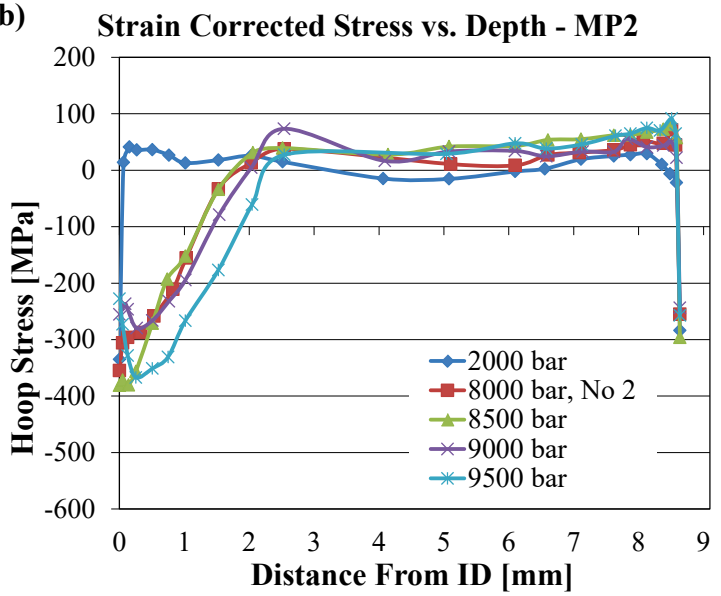

Fig. 4 - Hoop RS AF variation at MP2 - a) as measured and b) gradient, depth and strain corrected. 


\section{Conclusions}

In this paper, extensive XRD based RS measurements on AF thick walled cylinders were presented. The effects of pre-machining were found to be localized to the ID and OD surfaces and tended to result in a thin compressive RS layer. With the application of the AF process, significant compressive RS was imparted to the ID of the samples with concomitant balancing tensile RS near the OD. The general trend observed was an increase in the compressive RS layer with increasing AF pressure. An unanticipated result was observed in the curves for the specimens treated at higher AF pressures. It was expected that increasing the AF pressure would lead to a greater maximum magnitude of induced compressive RS. Instead, the apparent maximum magnitude of RS remained approximately the same with increasing AF pressure and in some cases, decreased. Nevertheless, the higher AF pressures produced a deeper compressive RS layer that was compensated for with higher magnitude subsurface tensile stresses. Post-machining, i.e. the reaming operation applied after the AF process which removes a part of the most compressive loaded material, led to a redistribution and relaxation of the balancing tensile RS towards the OD.

\section{References}

[1] Davidson, T.E., Barton, C.S., Reiner, A.N., Kendall, D.P., "The Autofrettage Principle as Applied to High Strength Light Weight Gun Tubes", U.S. Army T. R. WVT-RI-5907, Watervliet Arsenal, Watervliet, USA, 1959.

[2] H. Brünnet, M. Hofmann, N. Lyubenova, D. Bähre, Effect of Different Manufacturing Process Steps on the Final Residual Stress Depth Profile along the Process Chain of Autofrettaged ThickWalled-Cylinders, Proceedings of the $9^{\text {th }}$ European Conference on Residual Stresses, Advanced Materials Research (Volume 996), 2014, pp. 676-681. http://dx.doi.org/10.4028/www.scientific.net/amr.996.676

[3] Lechmann, M., "Development of a Fatigue Fracture Design Concept for Internal Pressure Loaded Parts with Extensive Compressive Stress Fields", Dissertation, Stuttgart University, Germany, 2008.

[4] Herz, E., Thumser, R., Bergmann, J.W., Vormwald, M., "Endurance Limit of Autofrettaged Diesel-engine Injection Tubes with Defects", Engineering Fracture Mechanics 73, 2006, p. 3. http://dx.doi.org/10.1016/j.engfracmech.2005.06.006

[5] H. Brünnet, D. Bähre, T. Rickert, D. Dapprich, Modeling and Measurement of Residual Stresses Along the Process Chain of Autofrettaged Components by Using FEA and Hole-Drilling Method with ESPI, Proceedings of the 9th International Conference on Residual Stresses, Materials Science Forum 768-769, 2014, pp. 79-86, http://dx.doi.org/10.4028/www.scientific.net/MSF.768-769.79

[6] Happe, U. "Entwicklungstendenzen im Hochdrudcbau", Chemie Ingenieur Technik, 24. Jahrg. 1952/Nr. 7, 1952, pp. 411-413.

[7] Brünnet, H.; Yi, I.; Bähre, D., "Modeling of Residual Stresses and Shape Deviations along the Process Chain of Autofrettaged Components", Journal of Materials Science and Engineering A, Volume 1/7A, 2011, pp. 915-936.

[8] Totten, G., Howes, M., Inoue, T., "Handbook of Residual Stress and Deformation of Steel, Chapter Measurement and Prediction of Residual Stress and Distortion", ASM International, 2002, pp. 112.

[9] Moore, M.G., Evans, W.P., "Mathematical Correction for Stress in Removed Layers in X-Ray Diffraction Residual Stress Analysis", SAE Residual Stress Committee, 1956.

[10] Ahmad, A., et. al., "Residual Stress Measurement by X-ray Diffraction", SAE HS-784, SAE International, Warrendale, PA, 2003, p.85. 